\title{
USO DE BIOMARCADORES CK-NAC, CK-MB E TROPONINA I EM CÃES COM DOENÇA CARDÍACA
}

(Biomarkers ck-nac, ck-mb and troponin I in dogs with heart disease)

\author{
Ariane da Costa Alves, Valéria Régia Franco Sousa, Erica Pereira da Silva, Pedro \\ Eduardo Brandini Néspoli, Felipe Gomes da Silva, Arleana Bom Parto Ferreira de Almeida \\ *Correspondência: ari_alves57@hotmail.com
}

RESUMO: O uso de biomarcadores cardíacos para diagnóstico clínico vem aumentando, porém, em medicina veterinária o uso ainda é restrito a pesquisas. Objetivou-se com esse trabalho avaliar a confiabilidade dos valores séricos de creatinoquinase, creatinoquinase fração MB e troponina I- fração cardíaca (Tnlc), na doença cardíaca correlacionando com a presença ou não de sinais clínicos. Foram avaliados 45 animais de diferentes raças, sexo e idade, separados em dois grupos. Ao grupo I foram selecionados 15 cães clinicamente sadios e sem alterações em radiografia, eletrocardiografia e ecocardiografia. No grupo II foram inseridos 30 cães com sinal clínico de doença cardíaca e com alteração em ao menos um dos exames utilizados na avaliação cardíaca. A idade variou de 1 a 11 anos de idade no grupo I (controle) e de 2 a 17 anos no grupo II. O sinal clínico mais relatado foi tosse, seguido por cansaço fácil. Os valores de referência para CK-MB no grupo I variou de 95 a 262 U/I com média de 157 $\pm 49,99$ e no grupo II (cardiopatas) variou de 74 a $787 \mathrm{U} / \mathrm{L}$ com média de $187,46 \pm 161,10$, porém não houve diferença significativa $(\mathrm{P} \leq 0,05)$ entre os grupos para os valores de CK-MB e CK-NAC. O teste rápido de troponina I - fração cardíaca (Tnlc) apresentou resultado negativo para os 45 animais do estudo. Nesta pesquisa, o CK-MB não se mostrou um bom indicador de doença cardíaca, porém recomendam-se análises futuras com aumento no número de cães.

Palavras-chave: cão; marcadores cardíacos; insuficiência cardíaca

\begin{abstract}
The use of cardiac biomarkers for clinical diagnosis has increased, however, in veterinary medicine the use still restricted to research. The aim of this study was to evaluate the reliability of serum creatine kinase values, creatine kinase MB fraction and troponin I-heart fraction (Tnlc) on heart disease correlated with the presence or absence of clinical signs. We evaluated 45 animals of different breeds, sex and age, divided into two groups. The group I have 15 healthy dogs without changes in radiography, electrocardiography and echocardiography. The group II was composed by 30 dogs with clinical signs of heart disease and change in at least one of the tests used for assessment. The age ranged from one to 11 years in group I (control) and 2-17 years in group II. The most frequent clinical sign was coughing, followed by tiredness. The reference values for CK-MB in group I ranged 95-262 U / I with an average of $157 \pm 49.99$ and in group II (heart disease) ranged 74-787 U / L with a mean of $187.46 \pm 16110$, but there was no significant difference $(p=0.05)$ between groups for CK-MB and CK-NAC values. The rapid test troponin I - Heart fraction (Tnlc) was negative for the 45 animals in the study. In this research, the CK-MB was not a good indicator of heart disease, but are recommended further analysis with an increase in the number of dogs.
\end{abstract}

Key Words: dog; heart markers; muscle-damage

Recebido em 31/01/2015

Aprovado em 21/08/2015 


\section{INTRODUÇÃO}

Estudos e pesquisas têm sido efetuados para averiguar a utilidade dos biomarcadores circulantes específicos de lesão e função cardíaca em Medicina Veterinária para melhorar os métodos de diagnóstico, monitoração ou mudança de terapias em pacientes com alterações cardiovasculares (Schober, 2010; Oyama, 2007; Terri, 2008; Yonezawa et al., 2009). De acordo com Schober (2010) e Terri (2008) estes biomarcadores demonstraram ser úteis na distinção de doença cardíaca e não cardíaca; lesão miocárdica e sua extensão associada a doença cardíaca isquêmica, drogas cardiotóxicas, trauma torácico, miocardite (Schober, 2010; Oyama e Sisson, 2007; Terri, 2008), ou ainda na doença cardíaca assintomática ou minimamente sintomática permitindo uma intervenção terapêutica mais precoce (Terri, 2008).

Para Ladenson (2007), o marcador cardíaco ideal precisa atender a alguns critérios, tais como: alta sensibilidade, sendo detectado precocemente mesmo em baixa quantidade em injúria miocárdica leve; alta especificidade, não sendo detectáveis em outros órgãos ou tecidos; ser facilmente quantificado e de baixo custo. Porém, nenhum dos marcadores cardíacos disponíveis atualmente apresentam todos os critérios.

Dois grupos de marcadores são descritos: os que indicam morte das células miocárdicas, os biomarcadores de lesão, como as troponinas cardíacas I e $T(c T n l / T)$, a mioglobina, a creatinoquinase $\mathrm{MB}(\mathrm{CK}-\mathrm{MB})$ e o lactato desidrogenase (LDH) (Terri, 2008), e aqueles liberados quando ocorre qualquer tipo de estresse patofisiológico, levando a insuficiência e disfunção cardiovascular. Estes últimos são normalmente marcadores neurohumorais ou inflamatórios (Boswood, 2007), como a proteína C- reativa, interleucina-6, peptídeos natriuréticos, entre outros.

O presente trabalho tem por objetivo avaliar a confiabilidade dos valores séricos de creatinoquinase, creatinoquinase fração $\mathrm{MB}$ e troponina Ifração cardíaca (Tnlc), na doença cardíaca correlacionando com a presença ou não de sinais clínicos.

\section{MATERIAL E MÉTODOS}

No presente estudo foram avaliados 45 cães, atendidos no HOVETUFMT de ambos os sexos, diferentes raças e idades. Os cães foram divididos em 2 grupos: Grupo I (controle) incluiu 15 cães, que não apresentaram alterações clínicas, como tosse, cianose, síncope, arritmia ou sopro, e alterações na radiografia torácica, ecocardiografia em modo $B$ e $M$, além de eletrocardiografia convencional, condizentes com doença cardíaca. Grupo II (cardiopatas) composto de 30 cães, os quais apresentaram alterações clínicas relacionadas a doença cardíaca e alteração em raio- $x$, eletrocardiograma ou ecocardiograma.

De todos os cães inseridos na pesquisa, foram coletados $5 \mathrm{~mL}$ de sangue por punção jugular, com prévia assepsia local. As amostras foram acondicionadas em frascos siliconados sem anticoagulante e centrifugadas a 3600 rpm por 5 minutos. O soro obtido foi dividido em duas alíquotas, armazenados em microtubos e uma mantida refrigerada em temperatura de $2-8^{\circ} \mathrm{C}$ por no máximo sete dias, e outra congelada a $-20^{\circ} \mathrm{C}$ para posterior análise.

A dosagem sérica de CK-NAC e CK-MB, foi realizada com as amostras mantidas refrigeradas, por meio de processo cinético utilizando kits comerciais, seguindo recomendações do fabricante. Para tal análise foi empregado analisador bioquímico semiautomático SB-190. A fração 
cardíaca (Tnlc) foi realizada empregando amostras mantidas congeladas, e realizada utilizando kit cromatográfico rápido, segundo recomendações do fabricante. Amostras hemolisadas e lipêmicas foram evitadas, a fim de não causar resultado falso positivo, em ambos.

Os dados obtidos foram tabulados e analisados estatisticamente utilizandose o software SAS 9.0, empregando-se os procedimentos GLM (análise de variância), REG (regressão linear nos parâmetros), MEANS (análises descritivas) e UNIVARIATE (testes de normalidade). Os níveis de significância considerados em todos os testes foram de $5 \%$ de probabilidade.

Os resultados dos testes de normalidade para as variáveis respostas, CK-MB e CK-NAC, indicaram que as mesmas não seguem distribuição normal dessa forma foram testadas diversas transformações de dados, obtendo bons resultados de normalidade para as transformações $z=1 / x$, em que $z$ representa a variável transformada e $\mathrm{x}$ a variável original. Já para a razão CK-MB e CK-NAC os resultados indicaram normalidade dos dados, sendo esta variável analisada sem sofrer transformação.

Para verificar a influência do efeito do animal ser cardíaco ou não (classificação por diagnóstico) sob os níveis das variáveis CK-MB e CK-NAC transformadas e a razão CK-MB e CKNAC, foram testados diferentes modelos reduzidos a partir do modelo completo que verificava a influência do diagnóstico (positivo ou negativo), sexo (masculino ou feminino) e idade do animal (efeitos linear e quadrático), conforme a equação:

$$
\begin{aligned}
y_{i j k}=\mu+d_{i} & +s_{j}+\beta_{1}\left(I_{k}-\bar{I}\right) \\
& +\beta_{2}\left(I_{k}-\bar{I}\right)^{2}+e_{i j k}
\end{aligned}
$$

$y_{i j k}=$ valor observado para a variável resposta (CK-MB ou CK-NAC);

$\mu=$ efeito médio incidente sobre todas as unidades experimentais; $d_{i}=$ efeito do diagnóstico cardíaco do animal i (positivo ou negativo);

$s_{j}=$ efeitos fixos do sexo j (macho ou fêmea);

$\beta_{1}=$ efeito linear da influência da idade sobre as variáveis respostas;

$\beta_{2}=$ efeito quadrático da influência da idade sobre as variáveis respostas;

$e_{i j k}=$ efeito aleatório residual incidente sobre cada unidade experimental.

\section{RESULTADOS E DISCUSSÃO}

Dos 15 cães do grupo I (controle) 11 eram fêmeas e quatro machos, já no grupo II (cardiopatas) dos 30 cães, 13 eram fêmeas e 17 machos. Porém, modelos completos e reduzidos não encontraram efeitos significativos para nenhuma das características classificatórias testadas (sexo e cardiopatia). Na avaliação clínica o sinal clínico mais relatado pelos proprietários nos cães do Grupo II (cardiopatas) foi tosse, presente em 21 (70\%) dos animais, seguido por cansaço fácil, presente em oito $(26,66 \%)$.

Neste estudo a idade variou de 1 a 11 anos de idade no grupo I (controle) e 2 a 17 anos de idade nos animais do grupo II (cardiopata) e segundo dados estatísticos a idade influenciou significativamente $(P<0.05)$ os valores de CK-MB e CK-NAC, apresentando efeito quadrático. Os resultados dos níveis de significância e parâmetros estimados para a equação de regressão estão expressos na Tabela 1. Tal observação pode ser explicada pela maior ocorrência de alterações cardíacas em animais idosos, excetuando as miocardiopatias de caráter congênito. Associado a isso, o diagnóstico de endocardiose de mitral em $10(33,33 \%)$ cães do grupo II reforçam tal análise, tendo em vista a maior ocorrência dessa afecção em cães com idade superior a sete anos (Borgarelli e Haggstrom, 2010). 
Tabela 1 - Resultados das equações de regressão para os valores de CK-MB e CK-NAC em função da idade dos cães.

\begin{tabular}{llll|}
\hline Característica & Parâmetro & Valor & Significância \\
\hline \multirow{2}{*}{ CK-MB } & Intercepto & 292,0095 & $<0,0001$ \\
& Linear & $-53,3627$ & 0,0022 \\
& Quadrático & 0,9476 & 0,0004 \\
\hline \multirow{3}{*}{ CK-NAC } & Intercepto & 107,3312 & 0,0005 \\
& Linear & $-16,6700$ & 0,0425 \\
& Quadrático & 1,0312 & 0,0305 \\
\hline
\end{tabular}

Lopes et al. (2005) descrevem que a Creatina Quinase (CK- NAC) é a enzima mais amplamente utilizada para determinação de doenças neuromusculares dos animais domésticos e que este é um indicador altamente sensível e específico de lesão muscular, já que os principais tecidos fonte dessa enzima são as fibras musculares esqueléticas, as cardíacas e ainda o músculo liso, predominando no tecido muscular esquelético e cardíaco. E ainda (Duncan et al., 1994; Cardinet, 1997; Kramer e Hoffmann, 1997) confirmam a presença de três isoenzimas da CK nos animais, como a isoenzima MM, encontrada em músculos esqueléticos e no miocárdio, a qual tem a maior vida média das isoenzimas de CK no cão; $M B$, também presente nesses mesmos músculos, porém em menor concentração; e BB, que é encontrada no tecido cerebral.

Os valores encontrados para CKNAC variaram de 26 a $307 \mathrm{U} / \mathrm{l}$, com média $63,73 \pm 68,62$ em animais sem sinais de cardiopatia (Grupo I) e de 19 a $219 \mathrm{U} / \mathrm{l}$, com média de 60,36 $\pm 51,09 \mathrm{em}$ cães com sinais de cardiopatia (Grupo II). Os valores mínimos e máximos encontrados nos cães do grupo I (controle) e nos cães do grupo II (cardiopatas) estão abaixo dos citados por (Duncan, 1994), cujo, intervalo de referência varia entre 52 a $368 \mathrm{U} / \mathrm{L}$.

Já os valores de CK-MB variaram de 95 a 262 U/I com média de 157士 49,99 nos animais sem sinais clínicos (Grupo I) e 74 a 787 U/l com média de 187,46 \pm 161,10 nos animais com sinais de cardiopatia (Grupo II), estando, portanto, todos os 45 cães desse experimento acima dos valores apresentados por Lopes et al (2005) onde os valores obtidos variaram de 11 a $38,8 \mathrm{U} / \mathrm{L}$ com média de $23,9 \pm 6,83$. Os dados obtidos aproximam-se dos encontrados por Athayde et al. (2014), onde a média dos valores de CK-MB em cães sem a presença sinais clínicos (Grupo I) e com a presença de sinais clínicos (Grupo II), encontrada foi de $200,35 \mathrm{U} / \mathrm{L}$ e 119,93 $\mathrm{U} / \mathrm{L}$ respectivamente. Os dados obtidos por Athayde et al. (2014), diferem dos comumente descritos, já que animais sem sinais de doença cardíaca apresentaram média dos valores de CKMB acima da média dos animais que não apresentaram sinais clínicos de doença cardíaca. No entanto, pode ser explicado em virtude da fração CK-MB também estar presente em outros órgãos como rim, intestinos e pulmões, não sendo, portanto, 100\% específica (Schober, 2010).

Os resultados encontrados para a razão CK-MB/CK-NAC não foram significativos $(p \leq 0,05)$. De qualquer forma, uma vez mensurada, é importante considerar os valores da CK-MB em relação à CK- NAC total para se determinar se está realmente aumentada. Ou seja, a porcentagem de CK-MB em relação ao valor total de CKNAC fornece um dado confiável quando se avalia uma possível lesão do miocárdio, pois valores aumentados de CK-MB acompanhados de aumento de CK-NAC podem ser resultantes de esforço físico, por exemplo. Mas, quando se observa aumento de CK-MB não acompanhado de aumento da CK-NAC total, a porcentagem dessa isoenzima fica elevada, comprovando-se lesão no músculo cardíaco (Melo et al., 2008). Com isso, dos 30 animais presentes no Grupo II (cardiopatas), 20 apresentaram 
valores de CK-NAC abaixo do valor de referência 52 U/L (Duncan, 1994), e desses 20, todos apresentaram valores de CK-MB acima do valor de referência 38,8 U/L (Lopes et al., 2005) e 11 apresentaram valores acima de $119 \mathrm{U} / \mathrm{L}$, (Athayde, 2014), podendo, portanto, esses animais, segundo (Melo et al., 2008) terem uma lesão no miocárdio. Porém, níveis de CK-MB para cães ainda não possuem valores de referências citados em literatura, seus valores estão restritos apenas em poucos artigos.

Todos os 45 animais que foram submetidos ao teste cromatográfico rápido de Troponina I, para verificar a presença ou não de infarto do miocárdio obtiveram resultados negativos. Segundo Costa et al. (2014) a Troponina I são específicas para lesão do miocárdio, principalmente infarto agudo, evento raro em cães, o que limita sua utilização na rotina veterinária, ficando restritas aos estudos experimentais. No entanto Oyama (2013) descreve aumento em pacientes portadores de doença cardíaca crônica, porém em magnitude inferior. A utilização, nesta pesquisa, de teste diagnóstico qualitativo para mensuração dessa isoenzima possa ter impossibilitado detectar aumento nos cães cardiopatas em comparação aos sadios, como observado Ljungvall et al. (2010)

\section{CONCLUSÃO}

Os resultados encontrados indicam que o CK-MB, mesmo acompanhado com valores de CK-NAC, não pode ser utilizado como indicador de doença cardíaca, uma vez que não houve diferença significativa entre os grupos avaliados. Em futuros trabalhos sugerese a inclusão do efeito da idade do animal como covariável linear e quadrática para avaliação do CK-MB/CKNAC e recomenda-se o aumento do número de unidades experimentais e testar a influência de outros efeitos como raça e peso.

\section{NOTAS INFORMATIVAS}

O presente estudo foi aprovado pelo Comitê de Ética no Uso de Animais (CEUA) da UFMT sob o número 23108.021280/13-0.

\section{REFERÊNCIAS}

ATHAYDE, L.C; FERNANDES, M.P.C; LEMOS, D.C. et al. Níveis séricos de creatinoquinase-MB em cães com e sem sinais de cardiopatia. Revista Nosso Clínico, p. 30-36. 2014.

BORGARELLI, M.; HAGGSTROM, J. Canine Degenerative Myxomatous Mitral Valve Disease: Natural History, Clinical Presentation and Therapy. Veterinary Clinics of North America: Small Animal Practice, v.40, p.651-663, 2010.

BOSWOOD, A. Laboratory tests for the diagnosis of heart disease and failure in dogs and cats. In: Proceeding of the World Small Animal Veterinary Association, Sydney, Australia 2007. Acesso em Novembro 2, 2014, disponível em:

www.ivis.org/proceedings/wsava/2007/pdf/81 20 070504082230 abs.pdf.

CARDINET, G.H. Skeletal muscle function. In: KANEKO, J.J.; HARVEY, J.W.; BRUSS, M.L. Clinical biochemistry of domestic animals. 5 ed. London: Academic Press, 1997. p 407-440.

COSTA, A.P.A.; NASCIUTTI, P.R.; DOS SANTOS JUNIOR, M.B.S. et al. Utilização de biomarcadores para avaliação da função Cardíaca em pequenos animais. Enciclopédia biosfera, Centro Científico Conhecer - Goiânia, v.10, n.18; p. 2989-3005, 2014.

DUNCAN, J.R.; PRASSE, K.W.; MAHAFFEY, E.A. Veterinary Laboratory Medicine - Clinical Pathology. 3 ed. lowa: lowa State University, 1994. 300 p.

ETTINGER, S.J.; FELDMAN, E.C. Tratado de medicina interna veterinária de pequenos animais: moléstias do cão e do gato. São Paulo: Manole, 2004.

KRAMER, J.W.; HOFFMANN, W.E. Clinical enzymology. In: In: KANEKO, J.J.; HARVEY, J.W.; BRUSS, M.L. Clinical biochemistry of domestic animals. 5 ed. London: Academic Press, 1997. p.303-325. 
LADENSON, J.H. A personal history of markers of myocyte injury [myocardial infarction]. Clinical Chimica Acta, v.381, n.1, p.3-8, 2007.

LJUNGVALL, I.; HOGLUND, K.; TIDHOLM, A.; OLSEN, L. H.; BORGARELLI, M.; VENGE, P. Cardiac troponina I is associated with severity of myxomatous mitral valve disease, age, and Creactive protein in dogs. Journal of Veterinary Internal Medicine, v. 153-159, 2010.

LOPES, S.T.A.; FRANCISCATO, C.; TEIXEIRA, L. V.; OLIVEIRA, T.G.M.; GARMATZ, B. C.; VEIGA, A.P.M.; MAZZANTI, A. Determinação da creatina quinase em cães. Revista da FZVA Uruguaiana, v. 12, n. 1, p. 116-122, 2005.

MELO, M.M.; VERÇOSA JÚNIOR, D.; PINTO, M. C.L.;SILVEIRA, J. B.; FERRAZ, V.; ECCO, R.; PAES, P. R. O. Intoxicação experimental com extratos de Mascagnia rigida (Malpighiaceae) em camundongos. Arquivo Brasileiro de Medicina Veterinária e Zootecnia, v. 60, n. 3, p. 631-640, 2008.

OYAMA, M.A. Cardiac blood tests: When, how and why to use them. In NAVC Proceedings 2007, North American Veterinary Conference, 13-Jan-2007. Disponível em: www.ivis.org/proceedings/navc/2007/

SAE/058.asp?LA=1. Acesso em 09/05/2014.

OYAMA, M.A.; SISSON, D.D.; SOLTER, P.F. Prospective screening for occult cardiomyopathy in dogs by measurement of plasma atrial natriuretic peptide, B-type natriuretic peptide, and cardiac troponin-I concentrations. American Journal of Veterinary Research, v.68, n.1, p. 42-47, 2007.

OYAMA, M.A. Using Cardiac Biomarkers in Veterinary Practice. Veterinary Clinics of North America: Small Animal Practice, v. 43, p. 12611272, 2013.

SCHOBER, K. Biochemical markers of cardiovascular disease. In: ETTINGER, S. J.; FELDMAN, E.C. Textbook of veterinary internal medicine. v. 2, 6. Ed. St. Louis, Missouri: Elsevier Saunders; 2010. p. 940-947.

SOLTER, P.F. et al. Canine heterophilic antibodies as a source of false-positive B-type natriuretic peptide sandwich ELISA results. Veterinary Clinical Pathology, v.37, n.1, p.8695, 2008.

TERRI, D.F. Cardiac biomarkers: A blood test for heart disease? In: Proceeding of the LAVC Latin American Veterinary Conference, Oct. 36, 2008, Lima, Peru. pp. 30-33.2008. Acesso em Novembro 2, 2014, disponível em: www.ivis.org/proceedings/

lavc/2008/francesco_terri.pdf.
YONEWAZA, L.A.; SILVEIRA, V.F.; MACHADO, L.P. et al. Marcadores cardíacos na medicina veterinária. Ciência Rural, v.40, n.1, p.222-230, 2010. 